\title{
Optimización de competencias de la trabajadora social en la Investigación Tutelar en el MIMP, UPE Lima-2015
}

Artículos originales: TRABAJO SOCIAL

Recibido: $10 / 09 / 2020$

Aprobado: 20/11/2020

Publicado: 05/02/2021

\author{
Carmen Violeta Vegas Cárdenas \\ Universidad Nacional Mayor de San Marcos \\ viovegas@hotmail.com
}

\begin{abstract}
RESUMEN
La presente investigación es producto de la experiencia profesional en la Dirección de Investigación Tutelar (hoy Dirección de Protección Especial) en el año 2015, donde se ha desempeñado la función de Trabajadora Social. De ahí que, los contenidos de la misma indagan sobre la influencia e importancia del aspecto social en la determinación e impacto de las medidas de protección hacia un niño, niña o adolescente en presunto estado de abandono, lo cual se plasma en la identificación de las competencias del trabajador social en este ámbito de acción. La teoría que guía los procedimientos y la metodología utilizada así como el análisis de la información responde a las ciencias sociales. Por ello, aspectos como la historia, la influencia económica y política, guardan estrecha relación cuando se evalúa la situación de la infancia, con sus problemas así como en sus soluciones.
\end{abstract}

Palabras Clave: Investigación tutelar; medidas de protección; desprotección; familia; competencias.

\section{Competencies Optimization of the Social Worker in Tutelary Research in the MIMP, UPE Lima-2015}

\begin{abstract}
This research is the product of professional experience in the Directorate of Tutelary Research (now the Directorate of Special Protection) in 2015, where the Social Worker function has been performed. Hence, its contents inquire about the influence and importance of the social aspect in the determination and impact of protection measures towards a child, girl or adolescent in an alleged state of abandonment, which is reflected in the identification of the Social worker competencies in this field of action. The theory that guides the procedures and the methodology used as well as the analysis of the information responds to the social sciences. Therefore, aspects such as history, economic and political influence are closely related when assessing the situation of children, with their problems as well as in their solutions.
\end{abstract}

KEYwORDs: tutelary research; protection measures; deprotection; family; competencies.

1 En diciembre del año 2016, el gobierno actual emitió el DL 1297, "Decreto Legislativo para la protección de nińas, niños y adolescentes sin cuidados parentales o en riesgo de perderlos", cuyo reglamento se publicó en el diario oficial El Peruano y entró en vigencia en febrero del 2018. Este trabajo se basa en la experiencia profesional del año 2015; es decir, cuando aún no se producía el cambio en el modelo de atención y por ende los términos: presunto estado de abandono, investigación tutelar, no se han modificado para efectos de la interpretación de la encuesta. 


\section{Introducción}

L a gran cantidad de niñas, niños y adolescentes (NNA) en nuestro país en presunto estado de abandono que se encuentran en las calles, en los Centros de Acogida Residencial: CAR (antes albergues) y viviendo en estado de explotación por personas que no son familiares, es un problema que nos demuestra que a pesar de los claros indicadores de desarrollo económico no se ha alcanzado igual mejora en el caso del desarrollo social y humano.

Sólo en el "Programa Integral Nacional para el Bienestar Familiar (INABIF), a nivel del país son casi 17,000 nińas, niños y adolescentes que se encuentran viviendo en los CAR existentes en casi todas las regiones" (DP 2011: 15) una cantidad alta sin considerar los centros privados. Otro aspecto del gran problema de estos NNA es que de ellos, sólo un $10 \%$ se han reinsertado a sus familias.

A nivel nacional es el Poder Judicial quien aún define la "suerte" de NNA en presunto estado de abandono. En Lima desde la creación de la Dirección de Protección Especial del Ministerio de la Mujer y Poblaciones Vulnerables (MIMP), son equipos interdisciplinarios quienes asumen, investigan y definen la situación de NNA dándole un tratamiento más técnico-administrativo y menos judicial (El Estado ha puesto como unos de sus propósitos desjudicializar el tratamiento a esta población).

A pesar de los cambios que en materia de niñez y adolescencia se han obtenido; no obstante el tema de la reinserción familiar que es el objetivo de las políticas sociales por la importancia que tiene la familia en la formación personalizada, estable y afectiva de esta población menor de 18 años, especialmente de los más pequeños, aún no se ha logrado una plena y mayoritaria integración familiar.

Los bajos porcentajes de NNA que se han reinsertado a un ambiente familiar tiene que ver con la permanencia de conceptos respecto a que es lo mejor para los NNA, a los procedimientos tradicionales a nivel de los operadores para tomar las decisiones más "rápidas", teniendo como la opción más "simple" trasladar a los CAR a todo NNA en presunto abandono y riesgo, con lo cual se termina la intervención referente a esta población infantil. Otro factor preocupante es la recarga de tareas administrativas, la cuantificación de metas de población atendida más que la calidad de la atención; y además el escaso compromiso de muchos de los profesionales encargados de trabajar con esta población. Otro aspecto que ha salido a la luz con motivo de la planificación por resultados, es la ausencia de mecanismos de seguimiento y retroalimentación con casos exitosos.

Nuestra propuesta desde el lado social es resaltar la importancia de las redes familiares y comunales en los casos de presunto abandono; y la consideración relevante de los aspectos sociales, económicos y culturales (de desarraigo, extrema pobreza y de violencia). Por ello un trabajo interdisciplinario y en conjunto con los sectores públicos y privados que tiene que ver con las condiciones económicas, de empleo, educación, salud y servicios en general que beneficia a los niños, niñas y adolescentes cobra mayor importancia.

\section{Situación problemática}

Debido a la gran cantidad de NNA albergados en los CAR que no logran ser reinsertados a su medio familiar y a los que se encuentran en presunto estado de abandono, cuyo número va en ascenso, se ha formalizado la creación de un área específica para que realice la investigación de esta población y se proceda a ejecutar las medidas de protección y el restablecimiento de sus derechos.

La Dirección de Protección Especial (DPE) es dependiente del Ministerio de la Mujer y Poblaciones Vulnerables (MIMP) y ha recibido un presupuesto especial en la modalidad de Presupuesto por Resultados para enfrentar el problema de esta población, en tiempo oportuno y con resultados visibles, uno de los cuales es la disminución de NNA institucionalizados en albergues.

La DPE funcionaba sólo en Lima, lo que hacia que se centralicen mucho los procedimientos legales y sociales; a partir del año 2013 se ha iniciado el proceso de desconcentración con la creación de las Unidades de Protección Especial (UPE) ubicadas en Junín, Arequipa, Cuzco, Madre de Dios, Piura, Loreto, Cajamarca, Lambayequ e, Tacna, Tumbes, Apurímac, Lima, Lima Este, Lima Norte (2015) y Lima Sur. Ello significa que será el ente rector quien asuma esta función de investigación que anteriormente, en el caso de las provincias estaba en manos del poder judicial. Aquí cabe preguntarse, qué es la investigación tutelar: 
Es el procedimiento de carácter mixto (administrativo y Judicial) a través del cual se realizan las diligencias necesarias que permitan restituir el ejercicio de los derechos de una nińa, nińo o adolescente en presunto estado de abandono, priorizando el de vivir con su familia y disponiendo las medidas de protección provisionales necesarias para su desarrollo integral. ("Decreto Supremo No 005-2016MIMP," 2016)

El trabajador social, es uno de los profesionales que participa en la investigación tutelar conjuntamente con un profesional de psicología y un abogado, pero es él quien realiza las visitas domiciliarias, las entrevistas, conoce la comunidad, la familia extensa, a los profesores,vecinos y autoridades de la localidad donde vivía el NNA. En caso de que el NNA se reintegre a su hogar el trabajador social diseñará medidas de asesoría y seguimiento a la familia para fortalecer los lazos y redes familiares y sociales de apoyo. Pese a este trabajo social y a la conformación de grupos multidisciplinarios en la DPE, los NNA nuevamente se fugan de su casa. $\mathrm{Al}$ parecer las investigaciones son insuficientes y no se tomó en cuenta algunos datos, como la persistencia y el grado de la violencia entre los padres y contra el NNA, la presencia del abusador en casos de violación sexual como las causas más frecuentes y que ponen en riesgo la integridad de los NNA.

\section{Formulando el problema de investigación}

La DPE no está logrando los resultados esperados respecto a la restitución del derecho de los NNA a vivir en familia. En ese contexto surge la interrogante respecto a: ¿La optimización de las competencias de la trabajadora social puede aportar a mejorar el papel de las UPE por tanto las decisiones en favor de los derechos del NNA?

\section{Justificación de la investigación}

La investigación puede señalar de manera más precisa los problemas de la investigación tutelar y aportar mejoras concretas al trabajo interdisciplinario, con ello se beneficiarán los NNA, sus familias y nuestro pais ya que se estará contribuyendo a disminuir niveles de violencia y a promover una cultura de paz.
Otra justificación de la importancia de la investigación es por su contribución a fortalecer personas más equilibradas y con dominio de sus emociones y constructoras de planes de vida que contribuyan a su desarrollo integral y al desarrollo humano de la localidad.

Los resultados de la investigación pueden aportar a la mejora de la formación profesional del trabajador social y al mejor funcionamiento de las UPE, las cuales pueden optimizar sus procedimientos. El papel del trabajador social es central, si éste logra dinamizar al equipo técnico articulando saberes y alternativas de seguimiento para la restitución de los derechos de los NNA obtendremos información más precisa en el diseño de un sistema de vigilancia social y monitoreo institucional lo cual puede disminuir los casos de reingreso y NNA en "situación de calle".

La investigación aportará a mejorar la formación académica de los trabajadores sociales y optimizará algunas funciones como la de investigación y gestión comunal, el manejo de las intervenciones sistémicas con la familia y fortalecimiento del trabajo grupal de los NNA en riesgo.

Como objetivo central de investigación se propone: Identificar competencias del trabajador social para que aporte al mejoramiento de la funcionalidad de las UPE en el restablecimiento de los derechos de los NNA. Como elementos complementarios se plantearon los siguientes objetivos:

- Clasificar las competencias del trabajador social en la investigación tutelar en el MIMP, UPE Lima-2015.

- Describir la función que desempeña el trabajador social en la investigación tutelar en el MIMP, UPE Lima-2015.

- Conocer qué figura familiar participa más en la investigación tutelar en el MIMP, UPE Lima-2015.

\section{Antecedentes de investigación}

El problema de los NNA en presunto estado de abandono ha pasado por diferentes etapas y enfoques, se pensaba que los niños muy pobres cuyas familias no podían mantenerlos debían ser derivados a los "albergues" o "puericultorios" para que sea el Estado quien se responsabilice de ellos. Los albergues eran ambientes grandes y con atención masiva. Ahora ha 
cambiado la concepción de atención privilegiando ambientes hogareños, casas individuales con una madre cuidadora que atiende de 6 a 8 NNA.

En estos tiempos se dice que la pobreza no es causa para que el niño sea separado de su familia, como tampoco son los problemas que surgen entre hijos adolescentes y padres muy autoritarios, una causa de recibir a los NNA que se fugan del hogar en los CAR porque no quieren ser controlados o castigados.

En la Convención de los Derechos del Niño y en el Código de los Niños y Adolescentes encontramos indicaciones respecto a que la pobreza no es causa de internamiento de los niños en las instituciones especializadas y que se brindará todo el apoyo a las familias y NNA para que resuelvan conflictos y problemas; entre ellos con terapias de familia, asesoría y monitoreo permanente. En estas acciones tiene valiosa intervención la familia extensa, los vecinos, los profesores, las DEMUNAS y la DPE.

A los NNA se les denominaba "menores" y cuando cometían actos ilegales se les consideraba delincuentes juveniles cuya medida al igual que los adultos era la cárcel. Ahora se dictan medidas de libertad provisional y consejerías permanentes para ir modificando algunas conductas de los NNA y sus familias, buscando apoyo grupal y económico para que no se profundice su pobreza y sus problemas familiares. Uno de los textos fundamentales para conocer los derechos de los NNA sin cuidados parentales es la Convención de los Derechos del Niño donde se refieren directamente a los NNA en estado de abandono. Los artículos referidos a esta problemática son: Artículo $4^{\circ}, 18^{\circ}, 20^{\circ}, 25^{\circ}, 27^{\circ}$ (UNICEF 2006).

Algunos autores: Mayda Ramos evalúa la situación de niños y adolescentes en los CAR y el papel de las instituciones que los albergan, así como las metodologías empleadas; Gianina Tapia, que aborda la problemática de atención de NNA en presunto estado de abandono en el marco de la desjudicialización; Vicente Espinoza, sintetiza la naturaleza del abandono y realiza la diferenciación entre la doctrina de la protección integral y la doctrina de la situación irregular.

\section{Metodología}

El estudio se realizó en el Ministerio de la Mujer y Poblaciones Vulnerables, estuvo compuesto por cua- renta trabajadoras sociales las mismas que pertenecían a los equipos de evaluación, desarrollo y soporte familiar de la Unidad de Protección Especial Lima.

La muestra fue seleccionada con antelación al proceso de bifurcación de la Dirección de Investigación Tutelar y la UIT Lima-Este (Dic. 2015); se aplicaron 23 encuestas a trabajadoras sociales en su conjunto, posteriormente se delimitó en tiempo y espacio la UIT a investigar, que en ese caso sería la UPE Lima, en donde se aplicó las 17 encuestas restantes. Es necesario mencionar aquí, que en cada Unidad de Investigación Tutelar (hoy UPE) laboran aproximadamente entre 15 a 18 trabajadoras sociales distribuidas en los equipos de evaluación, desarrollo, soporte familiar e itinerante. En cuanto a la tipología de investigación que se ha definido es descriptiva, diseño no experimental de corte transversal.

Para esta investigación se aplicó la encuesta, la cual se formuló en función de los objetivos de la investigación, por ello nos interesó saber las experiencias, grado de aprendizaje y reflexión en el campo de la DPE de las trabajadoras sociales, sus preconceptos o teoría personal que las guían y las razones que les hacen priorizar u ordenar sus acciones. Tratándose de la intervención de las trabajadoras sociales que son en su mayoría mujeres nos basaremos en la figura femenina.

En las preguntas de la encuesta nos interesó conocer cuáles eran los criterios profesionales y de sentido común que manejan estas profesionales para explicarse los fenómenos del abandono y que reflejan su marco conceptual y teórico desde el cual ordenan, priorizan u omiten algunas acciones respecto al abandono de los niños y niñas que atienden. De esa manera podemos saber hasta qué punto conviven y entran en tensión las concepciones asistencialistas reparadoras y de promoción de derechos; y cuáles serían las pistas para reforzar el segundo aspecto y qué necesitaríamos para ello. Es importante esta información para poder esbozar una propuesta de mejora viable desde el punto de vista social que refuerce la intervención profesional-institucional y de esta manera intervenir en el fortalecimiento de redes familiares y comunales.

Otro aspecto que se quiere recoger de la encuesta es el conocimiento que tiene la trabajadora social respecto al NNA en abandono y sus familias, así como sus experiencias positivas que han tenido en la DPE 
para poder compartir estos logros. Así mismo, queremos conocer los factores limitantes y errores para poder aprender de ellos y pasar al nivel de aprendizaje colectivo.

La otra finalidad de las encuestas es recoger la percepción de las trabajadoras sociales en cuanto a sugerencias y recomendaciones para tomarlas como referencias para mejorar la propuesta de la DPE, potenciando el aspecto social y familiar, que es lo que asume la trabajadora social para enriquecer el diagnóstico y diseñar pautas de trabajo para compartir con el equipo multidisciplinario promoviendo además el protagonismo de los NNA, sus familias y su comunidad.

Finalmente, respondiendo a los objetivos de la investigación y de la información proveniente de la encuesta que ayudará a replantear lineamientos que estén más relacionados con la restitución de derechos de los niños, niñas y adolescente en abandono que pueda realizar la DPE, y a partir de la influencia y aportes que ejerzan los trabajadores sociales los cuales potenciarán su perfil de intervención profesional, así como su renovación y reformulación de algunas de sus funciones tradicionales de asistencia social.

\section{Resultados}

La presente investigación pone énfasis en el enfoque social de la investigación tutelar, en la medida que es el trabajo primordial que realizamos como trabajadores sociales, el cual complementa y toma en cuenta el procedimiento legal y psicológico que se realiza en la intervención de los servicios de investigación tutelar. Resaltar el aspecto social es fundamental para la reinserción del NNA a su familia, o para hacer viable el Acogimiento Familiar del niño con personas que lo quieran y donde ellos se sientan bien.

El tratamiento social permite comprender las causas más comunes de la desprotección de los NNA, ya que en nuestro país tienen que ver mucho con problemas sociales, económicos y culturales de las familias de donde proceden los niños. Por lo mismo, la reintegración de su derecho a vivir en familia tiene que tomar en cuenta estos aspectos en su dimensión histórica y su contexto, para que las medidas que se adopten sean las mejores y sean sostenibles, es decir, modifiquen la situación de desamparo y descuido de
NNA y por lo mismo se les reintegre su derecho a vivir en familia.

Tener en cuenta los aspectos sociales que son fundamentales, así como también los procedimientos y protocolos de tipo normativo que son igualmente indispensables; la expectativa es que debieran influirse mutuamente enriqueciéndose además con la consideración de los daños y fortalezas a nivel psicológico y personal. Por ello, desde el inicio proponemos una articulación y sinergia entre todos estos elementos para irse recreando, modificando y haciéndose más aplicables y efectivos. La dimensión compleja del abandono o más bien de la desprotección infantil, obliga a comprenderla dentro de un contexto social, económico, cultural e histórico particularizando los diferentes casos que se presentan.

TABla 1. Parentesco de la persona que asiste a las citas del servicio de investigación tutelar

Personas que más acuden a las entrevistas en los casos de presunto estado de abandono del NNA

\begin{tabular}{|c|c|c|c|c|}
\hline & & Frecuencia & $\begin{array}{c}\% \\
\text { válido }\end{array}$ & $\begin{array}{c}\% \\
\text { acumulado }\end{array}$ \\
\hline & -Madre & 20 & 50,0 & 50,0 \\
\hline & -Padre & 4 & 10,0 & 60,0 \\
\hline & -Ambos padres & 4 & 10,0 & 70,0 \\
\hline$\stackrel{0}{=}$ & -Un hermano & 4 & 10,0 & 80,0 \\
\hline$i$ & -Abuela materna & 5 & 12,5 & 92,5 \\
\hline & $\begin{array}{l}\text { Representante de } \\
\text { institución }\end{array}$ & 3 & 7,5 & 100,0 \\
\hline & Total & 40 & 100,0 & \\
\hline
\end{tabular}

Fuente. Vegas, C. (2019)

Uno de los principales hallazgos es justamente la presencia de la madre y de la familia materna en la mantención y crianza de los hijos ( $50 \%$ y $12,5 \%$ respectivamente); así como en el interés por acercarse a las entrevistas y de salir y atender cuando se realizan las vistas domiciliarias. Cuando la madre tiene problemas de tiempo debido a sus largas jornadas de trabajo o de viajes por la misma razón, es la familia materna quien se encarga de acercarse a responder ante un presunto abandono. La mujer tiene una gran presencia en los hogares porque son ellas quienes salen a trabajar para que su familia se alimente y además asumen su cuidado y protección. Las situaciones de trabajos arduos y grandes distancias al movilizar- 
se, además de las jornadas al interior de las familias, obliga a mirar los problemas de las familias pobres en toda su complejidad, las cuales no se remedian con medidas únicamente de carácter legal que puedan surgir de algunos casos de presunto abandono; por el contrario su dimensión compleja obliga a comprenderla y trabajarla dentro de un contexto social, económico, cultural e histórico en particular, es decir, a nivel país (región, provincia, distrito).

Por lo tanto, tenemos que las colegas de trabajo social manifiestan que las personas que más asisten a las entrevistas y retornan y atienden en las visitas domiciliares son las madres o los familiares maternos, lo que se da en diferentes contextos limeńos o en provincias. Este hallazgo, obliga a trabajar de manera temprana e intersectorial políticas de Estado que fortalezcan la paternidad y el intercambio de roles en las familias, para incentivar la asunción de las responsabilidades del varón en relación con sus hijos e hijas.

Tabla 2. Causas de la desatención de los NNA

\begin{tabular}{|c|c|c|c|c|}
\hline \multicolumn{4}{|c|}{ Problemas más recurrentes con la familia del NNA } & \multirow[b]{2}{*}{$\begin{array}{l}\% \text { acu- } \\
\text { mulado }\end{array}$} \\
\hline & & Frecuencia & $\begin{array}{c}\% \\
\text { válido }\end{array}$ & \\
\hline \multirow{4}{*}{$\frac{0}{\frac{0}{7}}$} & $\begin{array}{l}\text {-Padre y madre no } \\
\text { asumen rol protector }\end{array}$ & 22 & 55,0 & 55,0 \\
\hline & $\begin{array}{l}\text {-Parientes no quieren } \\
\text { responsabilizarse }\end{array}$ & 14 & 35,0 & 90,0 \\
\hline & $\begin{array}{l}\text {-No tiene familia ni } \\
\text { conoce a nadie en la } \\
\text { ciudad }\end{array}$ & 4 & 10,0 & 100,0 \\
\hline & Total & 40 & 100,0 & \\
\hline
\end{tabular}

Fuente. Vegas, C. (2019)

Son diferentes causas por las que un padre o madre "no quieren" asumir su responsabilidad frente a su hijo (55,0\%). A nuestro entender y basándonos en la atención de casos, uno de los principales problemas es el conductual, no saber o más bien el no poder lidiar con un comportamiento ya formado o en proceso de formación pero con gran influencia de sus pares; en donde el proceso de socialización en la crianza de los hijos fue disfuncional o simplemente no hubo un modelo que emular.

Es realmente chocante escuchar a un padre y/o familia extensa decir que prefieren ver a su hijo en un albergue que junto a ellos, y revertir esta situación conlleva apuntar a trabajar en la concientización de la familia para la aceptación del niño en el retorno a su hogar; pero este proceso implica tiempo y mientras tanto nos preguntamos cómo queda ese nińo; como alternativa temporal recluirlo en un Centro Preventivo de la PNP que se traduce para la población adolescente en privación del derecho a la libertad, hacinamiento, malos tratos, retraso escolar y demás factores negativos. Es importante señalar que en el tiempo laborado en el MIMP (más de un año) ninguna familia mencionó el tema económico como causal de abandono, siendo familias con pobreza y pobreza extrema; pero resulta "cómodo" para ellos no asumir su responsabilidad y dejar que el Estado reemplace su función protectora, teniendo resuelto el tema de alimentación, educación, vivienda y recreación de sus hijos e inclusive sugiriendo al equipo técnico el tiempo de internamiento del niño.

TABLA 3. Instituciones a las que recurre el trabajador social

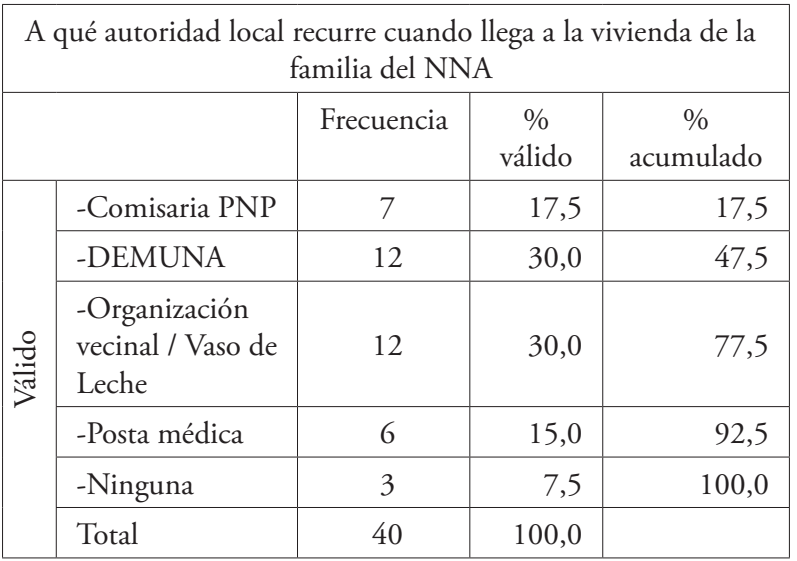

Fuente. Vegas, C. (2019)

Otro de los hallazgos importantes de la presente investigación es la presencia activa de las organizaciones sociales en el barrio y en la vida de las familias $(30,0 \%)$ que tienen problemas o situaciones producidas por la omisión, ejercicio inadecuado o incumplimiento de deberes hacia sus hijos. Los vecinos cercanos, la dueña de la bodega y principalmente las dirigentes del Vaso de Leche o Comedores Populares (a veces las iglesias) conocen los problemas que pasan los niños en mención, cuando se realizan visitas están enterados y brindan información importante lo que es muy útil para conocer más al detalle aspectos de la vida familiar. Esto nos indica que las redes socia- 
les están activas y constituyen un capital valioso que explotar.

Una segunda institución de ayuda en el seguimiento de casos son las DEMUNAS 30\%; es importante resaltar aquí el "Plan de Incentivos a la Mejora de la Gestión y Modernización Municipal"- Meta 20, que es la intervención de la DEMUNA en los casos de presunto abandono; la ejecución de las "[...] visitas domiciliarias en el marco del seguimiento a los planes individuales dispuestos por los equipos de la Dirección de Investigación Tutelar [...] lo cual permite vigilar que niñas, niños y adolescentes se reinserten en familias con condiciones que faciliten su protección” (MIMP 2015: 3). Por lo tanto, ambas instituciones fortalecen su accionar y se visibiliza a nivel poblacional la presencia institucional.

TABla 4. Problemas más importantes que tienen las trabajadoras sociales en las UIT

\begin{tabular}{|r|l|r|r|r|}
\hline \multicolumn{3}{|c|}{ Problemas en el trabajo organizacional de investigación tutelar } \\
\hline \multicolumn{2}{|c|}{} & Frecuencia & $\begin{array}{c}\% \\
\text { Válido }\end{array}$ & \multicolumn{1}{c|}{$\begin{array}{c}\% \\
\text { acumulado }\end{array}$} \\
\hline & $\begin{array}{l}\text {-No trabajan } \\
\text { colectivamente }\end{array}$ & 5,0 & 5,0 \\
\hline $\begin{array}{l}\text {-Sobrecarga del } \\
\text { trabajo }\end{array}$ & 25 & 62,5 & 67,5 \\
\hline $\begin{array}{l}\text {-Falta de } \\
\text { comunicación }\end{array}$ & 4 & 10,0 & 77,5 \\
\hline $\begin{array}{l}\text {-Recursos } \\
\text { humanos y } \\
\text { movilidad } \\
\text { limitados }\end{array}$ & 9 & 22,5 & 100,0 \\
\hline Total & 40 & 100,0 & \\
\hline
\end{tabular}

Fuente. Vegas, C. (2019)

En esta parte del análisis y, de acuerdo a los resultados obtenidos, vamos a enfatizar los problemas de la parte administrativa en la investigación tutelar que son comunes y se repiten de forma constante en los equipos multidisciplinarios.

El principal problema a destacar con un $62,5 \%$ es la sobrecarga de trabajo; si bien es cierto laborar en la DIT (hoy UPE) como profesional es satisfactorio y gratificante, pues compensa todo el esfuerzo grupal al ver restablecidos los derechos de niños y/o adolescentes es necesario resaltar aquí la otra cara de la moneda, como es la excesiva carga de expedientes por grupo, cabe preguntarse entonces: cómo ejecutar un correcto seguimiento de un caso " $\mathrm{x}$ " para llegar a buen puerto si es que tenemos acumulación de expedientes; reingresos de niños y/o adolescentes puestos a disposición por la Policía Nacional o familiares (que se tiene que "solucionar" el mismo día); así como la elaboración del Plan de Trabajo Individual (para su elaboración hay que leer todo el expediente) que establece los objetivos, estrategias, metas y plazos para disminuir o eliminar factores de riesgo y demás funciones por mencionar que realiza un trabajador social y, si sumamos a esto el problema número dos en importancia, según datos de la encuesta, en donde el $22,5 \%$ expresa que los recursos humanos y movilidad son limitados; tenemos pues, todo un engranaje de situaciones que hace que el profesional de la DIT se vea recargado en su trabajo, estresado y como consecuencia que realice una intervención repentizada y no planificada.

TABLA 5. Acciones o estrategias que aplica cuando surgen problemas

\begin{tabular}{|c|c|c|c|c|}
\hline \multicolumn{5}{|c|}{ Propuestas para mejorar el trabajo en equipo } \\
\hline & & Frecuencia & $\begin{array}{c}\% \\
\text { válido }\end{array}$ & $\begin{array}{c}\% \\
\text { acumulado }\end{array}$ \\
\hline \multirow{5}{*}{ 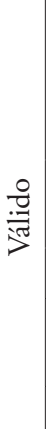 } & $\begin{array}{l}\text {-Promover } \\
\text { reuniones, } \\
\text { acuerdos, } \\
\text { consensos. }\end{array}$ & 21 & 52,5 & 52,5 \\
\hline & $\begin{array}{l}\text {-Concertar } \\
\text { protocolo }\end{array}$ & 14 & 35 & 87,5 \\
\hline & -Otros & 3 & 7,5 & 95 \\
\hline & $\begin{array}{l}\text {-No sabe /No } \\
\text { contesta }\end{array}$ & 2 & 5 & 100,0 \\
\hline & Total & 40 & 100,0 & \\
\hline
\end{tabular}

Fuente. Vegas, C. (2019)

Igualmente que en la tabla 4 vamos a enfatizar las acciones o estrategias de la parte administrativa de la investigación tutelar. Estando las trabajadoras sociales conscientes que podrían mejorar sustantivamente su trabajo si realizan una planificación participativa, realizan un seguimiento y reflexión de manera permanente, ellas contestaron en la encuesta de la siguiente manera: $52,5 \%$ proponen promover reuniones, acuerdos y consensos; frente a un escaso trabajo corporativo o colectivo, pues existen pocos espacios de encuentro donde se pueda planificar acciones de manera más seguida y enmendar limi- 
taciones o errores en el accionar, se ejerce más bien un desempeño individualizado a nivel de equipo, en donde las inquietudes y preguntas "para solucionar obstáculos" no cuentan en este sentido; por consiguiente no siguen la misma lógica cuando hacen los procedimientos, no reflexionan colectivamente, por lo cual no generan aprendizajes colectivos.

Entonces tenemos que la mayoría de trabajadoras sociales indica que esta situación se puede cambiar, generando espacios de debate y discusión, presentando casos-problemas, invitando a otros profesionales especialistas en el tema de infancia quienes tienen la capacidad de sensibilizar y llamar a la reflexión-acción. Entonces, el resultado obtenido será una cadena de valor de un trabajo articulado el cual produce grandes sinergias que contribuye a alcanzar el logro de los objetivos a nivel institucional y la reinserción familiar a nivel poblacional.

TABla 6. Limitaciones del trabajador social en la reinserción familiar

\begin{tabular}{|c|c|c|c|}
\hline \multicolumn{4}{|c|}{ Cuál es la mayor limitación que ha tenido en su trabajo } \\
\hline & Frecuencia & $\begin{array}{c}\% \\
\text { válido }\end{array}$ & $\begin{array}{c}\% \\
\text { acumulado }\end{array}$ \\
\hline $\begin{array}{l}\text {-No hay } \\
\text { vacante en } \\
\text { CAR }\end{array}$ & 13 & 32,5 & 32,5 \\
\hline $\begin{array}{l}\text {-Familia no } \\
\text { quiere tener a } \\
\text { NNA }\end{array}$ & 18 & 45,0 & 77,5 \\
\hline $\begin{array}{l}\text {-No hay } \\
\text { CAR para } \\
\text { NNA con } \\
\text { discapacidad }\end{array}$ & 3 & 7,5 & 85,0 \\
\hline $\begin{array}{l}\text {-Sobrecarga de } \\
\text { trabajo }\end{array}$ & 6 & 15,0 & 100,0 \\
\hline Total & 40 & 100,0 & \\
\hline
\end{tabular}

Fuente. Vegas, C. (2019)

Las trabajadoras sociales entrevistadas refieren que el problema más grande que enfrentan al realizar la investigación tutelar es la no aceptación del niño en el retorno a su hogar $(45,0 \%)$. Las razones principales que justifican esta negativa son porque cogen el poco dinero del que disponen en su casa o sustraen cosas para venderlas, retornan a la casa ebrios y arman peleas, vuelven a altas horas de la noche, "ya no obedecen” no hacen nada, no ayudan y se ponen atre- vidos con su familia, además porque ponen en peligro la vida de sus hermanos menores al llevarlos consigo constituyendo un mal ejemplo para ellos. Otra causa para no querer tenerlos de vuelta en familia es porque la casa se vuelve insegura por el tipo de amistades que tienen o frecuentan y la falta de respeto a las personas que viven en ese hogar. Se ha observado que un motivo fundamental que no lo manifiestan en las entrevistas pero que suele presentarse, sobre todo en los casos de reingreso o retorno de los NNA, es el beneficio económico que obtienen los familiares ya que las instituciones cubren todos sus gastos de alimentación, ropa, educación, salud y otros.

El segundo motivo en importancia que limita su trabajo de investigación tutelar tiene que ver con la ausencia de vacantes en los centros de atención residencial (32,5\%), debido a la sobrepoblación existente. Esta respuesta llama la atención porque significa que se sigue pensando en el internamiento como una alternativa común y que se mantiene como la acción más frecuente e inmediata. Para lograr la reinserción o acogimiento familiar supone más visitas, sensibilización, información, consejería permanente, seguimiento y supervisión.

TABla 7. Competencias que debe tener un trabajador social en la investigación tutelar

\begin{tabular}{|c|c|c|c|c|}
\hline \multicolumn{5}{|c|}{ Clasificación de competencias del trabajador social } \\
\hline & & Frecuencia & $\begin{array}{c}\% \\
\text { válido }\end{array}$ & $\begin{array}{c}\% \\
\text { acumulado }\end{array}$ \\
\hline \multirow{5}{*}{$\frac{?}{\frac{7}{i \pi}}$} & $\begin{array}{l}\text {-Competencia } \\
\text { técnica } \\
\text { (conocimientos) }\end{array}$ & 7 & 17,5 & 17,5 \\
\hline & $\begin{array}{l}\text {-Competencia } \\
\text { metodológica } \\
\text { (procedimientos) }\end{array}$ & 9 & 22,5 & 40,0 \\
\hline & $\begin{array}{l}\text {-Competencia } \\
\text { social } \\
\text { (formas de } \\
\text { comportamiento) }\end{array}$ & 11 & 27,5 & 67,5 \\
\hline & $\begin{array}{l}\text {-Competencia } \\
\text { participativa } \\
\text { (formas de } \\
\text { organización) }\end{array}$ & 13 & 32,5 & 100,0 \\
\hline & Total & 40 & 100,0 & \\
\hline
\end{tabular}

Fuente. Vegas, C. (2019)

Antes de empezar a explicar el cuadro de la clasificación de competencias del trabajador social, es 
necesario definir y tener en claro qué entendemos por competencias profesionales. Según el Prof. Dr. Gerhard Bunk (1994) "posee competencia profesional quien dispone de los conocimientos, destrezas y aptitudes necesarios para ejercer una profesión, puede resolver los problemas profesionales de forma autónoma y flexible, y está capacitado para colaborar en su entorno profesional y en la organización del trabajo" (p. 9). Partiendo de esta conceptualización tenemos que, un profesional sea cual fuese la profesión que ejerza tiene que estar en constante capacitación, es decir, una continua y persistente adquisición de conocimientos pues las transformaciones sociales, económicas y políticas del ámbito laboral y a nivel país así lo demandan. Pero estas competencias deben traspasar la simple especialización técnica y formal de los profesionales, se requiere incorporar dimensiones relacionales y sociales que se vean reflejadas en la capacidad real para la resolución de situaciones y problemas en donde la capacidad adquirida forma parte de un continuo proceso de aprendizaje y experiencia profesional.

Ahora bien, pasemos al análisis de la tabla 7; siendo la pregunta de la encuesta de tipo abierta, las colegas de trabajo social respondieron diferentes competencias para el ámbito de la investigación tutelar, las cuales se ordenaron y agruparon en una lista y para que la información brindada tenga sustento teórico, sea manejable al momento de tabular y analizar y por último sea comprensible para el lector; se las ubicó dentro del modelo de competencias de Bunk, uno de los autores más referenciados en el estudio de las competencias, quien clasifica las competencias en cuatro categorías: competencias técnicas, competencias metodológicas, competencias sociales y competencias participativas; en donde cada una de ellas tiene un contenido propio; las respuestas de las colegas se adaptaron a dicha categorización. A continuación se van a desarrollar las definiciones de éstas categorías de competencias:

Competencia técnica: Bunk (1994) indica que, posee "competencia técnica aquel que domina como experto las tareas y contenidos de su ámbito de trabajo, y los conocimientos y destrezas necesarios para ello" (p. 10). Por consiguiente, si trasladamos la información proporcionada por la encuesta obtenemos un conjunto de competencias que según ésta definición serían las siguientes: Capaz de identificar problemas,
Capaz de plantear preguntas con claridad, Realizar diagnósticos, Aptitudes para redactar informes, Está al día de la legislación vigente, Conocimiento de los recursos locales, Educación social y atención a la infancia, Orientación y promoción familiar, Violencia familiar y Gerencia social.

Competencia metodológica: Bunk (1994) seńala, implica la capacidad "[de] reaccionar aplicando el procedimiento adecuado a las tareas encomendadas y a las irregularidades que se presenten, que encuentra de forma independiente vías de solución y que transfiere adecuadamente las experiencias adquiridas a otros problemas de trabajo" (p.10). Algo semejante sucede con: Capacidad para recopilar, analizar y entender la información, Capaz de interpretar situaciones, Habilidad para gestionar conflictos, Habilidad para resolver problemas, Observador y Objetivo.

Competencia social: Bunk (1994) añade, implica saber "colaborar con otras personas de forma comunicativa y constructiva, y muestra un comportamiento orientado al grupo y un entendimiento interpersonal" (p. 10-11). Cosa parecida sucede también con: Capacidad para trabajar en equipo, Capaz de mantener la calma bajo presión, Actitud positiva, Trabaja en horas fuera del horario habitual, Perseverante, Resistente, Tolerante, Amable, Iniciativa y Sentido del humor. Por ejemplo, en la dinámica funcional interna de los equipos multidisciplinarios de las UPE; tanto trabajadores sociales, abogados y psicólogos tienen que necesariamente "entenderse", trabajar coordinadamente, en constante comunicación, esa es la clave del éxito para la productividad del equipo y la resolución de casos. Aquí, el trabajo sesgado por especialidad profesional o las relaciones interpersonales "tensas" entre profesionales, simplemente no funciona.

Competencia participativa: Bunk (1994) sostiene que, posee tal competencia "aquel que sabe participar en la organización de su puesto de trabajo y también de su entorno de trabajo, es capaz de organizar y decidir, y está dispuesto a aceptar responsabilidades" (p. 11). De igual modo tenemos: Organizado, Responsable, Capacidad para gestionar carga de trabajo, Capacidad de llevar varios casos de forma simultánea, Capaz de enfrentar situaciones difíciles, Capaz de tratar con personas difíciles o demandantes, Capaz de trabajar con personas con disminuciones psíquicas, Capacidad para tomar decisiones, 
Aptitudes para gestionar el tiempo, Resolutivo, Resuelto, Comprometido y Capacidad para negociar. En suma, afirmamos que estas cuatro competencias no actúan aisladamente, con independencia propia; sino todo lo contrario, éstas en su conjunto interactúan y se complementan en el quehacer profesional del trabajador social.

\section{Discusión}

1. El papel central de la figura materna, la ausencia del padre y por tanto la sobrecarga de trabajo de la madre como responsable de la familia sobre todo en los hogares pobres. A nivel de la teoría social sabemos que los hogares monoparentales son una realidad que se hace generalizable en muchos lugares del Perú (Muñoz, I. (Ed.).(2014), la historia, las diferenciaciones de género y la percepción de la paternidad que se vive en el ámbito de las masculinidades en detrimento de las mujeres en general y especialmente cuando son madres.

En este estudio pudimos ampliar esta idea referida a la investigación tutelar, por tanto sugerir que acciones se pueden enfatizar o modificar teniendo en cuenta como se presenta esta problemática en NNA en presunto estado de abandono. Por ejemplo poner especial interés en localizar al padre y no sólo trabajar con las madres.

De este hallazgo se desprende también la importancia de trabajar preventivamente desde las instituciones educativas de manera temprana, el intercambio de roles y responsabilidades en el hogar sobre todo referidos a la crianza de los hijos e hijas, brindando consejería a la madre y especialmente al padre cuando el NNA están atravesando problemas.

Esta idea se convierte en hallazgo cuando al interior de la investigación tutelar notamos la presencia especialmente de las madres que acuden a las citas o vienen a las entrevistas (50\%) o son las que deciden qué medidas tomar con sus hijos y al mismo tiempo -y paradójicamente- son las más ausentes cuando se trata de reinsertar nuevamente a la familia a los NNA (55\%). Constituyendo este hecho en uno de los principales problemas que enfrenta el trabajador social.

Los cuadros que vienen a continuación nos señalan las causas del primer hallazgo respecto a tener a la madre como figura central en la problemática del abandono infantil, afirmación preocupante para la sociedad y el Estado en general debido a las limitaciones y peligro de hacer descansar toda la responsabilidad en las mujeres las cuales además de engendrar y trabajar sin descanso son únicas responsables de todo lo que acontezca a sus hijos e hijas debido a la figura ausente del padre (10\%).

2. La importancia del enfoque intercultural. Este enfoque es importante ya que nos va a explicar porque hay mayor violencia y migración de las personas que vienen de los lugares donde fue mayor la violencia política y donde eran quechuahablantes o su gran exclusión. En el caso de la investigación tutelar el enfoque intercultural se traduce en el trato que debe existir, cálido y expresando visiblemente empatía como darles la mano, cambiando el tono de voz como hacen las personas en su entorno familiar.

La interculturalidad se manifiesta en una comprensión especial respecto a los problemas que atraviesan las personas de ámbitos rurales cuya explicación o razones podrían no ser comprendidas por nosotros. Por ejemplo la concepción respecto a la responsabilidad temprana que debe asumir un niño o niña, o la comprensión más metafórica o simbólica que tienen a diferencia del conocimiento cognitivo occidental.

Eso significa que nuestras preguntas, asesorías, indicaciones, comprensión de las cosas tiene que tener otro formato, evitando largos discursos, evitando términos técnicos o dando por hecho que ya comprendieron los trámites o los problemas que deben de asumir. Según Ansión y Tubino (2007) —en la bibliografía que ya dimos a conocer- asumir un enfoque intercultural significa cambiar nuestro sistema de valoración, reconociendo que la lógica occidental no es superior ni mejor a la que usan personas que pertenecen a culturas andinas o amazónicas, es decir que son diferentes, pero iguales en importancia. Esto supone, dicen los autores; funcionarios sensibilizados, conocedores y comprometidos con un cambio en la manera de pensar y de hacer las cosas. Y nos referimos con ello a los directores nacionales, fiscales, jueces y profesionales que trabajan en las UPE.

3. El trabajo interdisciplinario es una condición básica para hacer más efectiva la intervención del servicio de investigación tutelar, ya que los saberes se complementan y se tiene una comprensión más amplia e in- 
tegral de la situación actual de un NNA. El problema de la desprotección infantil en países como el nuestro con altos niveles de exclusión, violencia y pobreza; se constituye un problema complejo y multifactorial, donde interviene la condición económica de bajos ingresos, la exclusión a la que hacíamos mención y a la "cultura" o mentalidad que se tiene del niño o niña debido a su condición de inferioridad e incapacidad referida y al trato que debiera recibir un niño o nińa. Con acierto los trabajadores sociales afirman que buena parte de los problemas se disminuirían si el trabajo interdisciplinario fuera más eficaz.

4. La intervención del trabajador social tiene como principales funciones participar activamente en el diagnóstico de la situación actual de un niño, resaltando el contexto familiar y social donde vive, para saber cómo esos elementos influyen en su comportamiento. Además desde ese conocimiento proponer acciones para diseñar la evaluación interdisciplinaria y el plan de trabajo individual del NNA. Parte de sus funciones como señalan las mismas profesionales es aportar con propuestas para el acogimiento desde la recuperación de las redes familiares, sociales y comunales. Parte de la propuesta es que el MIMP se convierta realmente y de manera creciente en el órgano rector.

Debido a las crecientes atribuciones que van teniendo los gobiernos locales referidos al tema familiar y desarrollo humano e infancia y a la formulación de programas específicos desde el Ministerio de Economía y Finanzas con financiamiento y resultados específicos; se espera incorporar como parte de la responsabilidad de los NNA en presunto abandono o desprotección a los gobiernos locales distritales, provinciales y también a los gobiernos regionales. Lo que exige profundizar la transferencia de programas sociales y la modernización del Estado.

Mejorar la coordinación y el servicio del Ministerio Público y Poder Judicial; la información que damos a conocer en esta investigación corresponde a profesionales de trabajo social, cada una de las cuales atienden en un servicio de investigación tutelar y tiene que entablar acciones a veces problemáticas con estas instancias en lugar de que sea un trabajo complementario y cooperativo. Esto logramos definir de sus respuestas las cuales expresan las experiencias y aprendizajes de su labor durante 2 años en promedio, expresa la necesidad de promover canales más agiles para el trabajo de investigación tutelar. Lograr estrategias exitosas para el acogimiento familiar evitaría ciertamente el trabajo engorroso con establecimientos del Ministerio Público y Poder Judicial.

Un hallazgo importante frente a la información y aprendizaje de los trabajadores sociales es la identificación y clasificación de las competencias específicas de este profesional en el ámbito de la investigación tutelar, plasmadas en cuatro categorías: Competencia Técnica 17.5\%, Competencia Metodológica 22,5\%, Competencia Social 27,5 y Competencia Participativa 32,5.

\section{Conclusiones y recomendaciones}

1. El diseño de los programas sociales referidos a la investigación tutelar de las nińas, niños y adolescentes sin cuidados parentales o en riesgo de perderlos, tiene que ver con una nueva comprensión de la infancia como sujetos de derechos, de esta manera los NNA en presunto estado de abandono o desprotección familiar, no deben ser ubicados como NNA en situación irregular, producto de un ambiente familiar disfuncional, sino como NNA cuyos derechos han sido vulnerados así como los de su familia a donde no han llegado las oportunidades, la protección e impulso del Estado en general.

2. El problema de los niños y adolescentes desprotegidos es parte de los niveles tan bajos que tiene el Perú referentes al desarrollo humano, a pesar que hay indicadores de crecimiento económico, estos no han repercutido en mejorar las condiciones de vida de las familias más pobres. Son las familias monoparentales donde la mujer trabaja todo el día, las que tienen mayores problemas con los NNA, ya que estos salen a la calle en busca de compañía y de espacios de distracción que les hagan olvidar las privaciones y ambientes conflictivos que viven a diario.

3. Trabajar con niñas, niños y adolescentes desprotegidos en Lima y en las ciudades donde se han iniciado las UIT (hoy UPE), exige a las instituciones y profesionales conocer más el contexto socioeconómico y ahondar en aspectos históricos y culturales de las familias. Muchas de las familias que tienen problemas con sus hijos e hijas 
son de condición pobre y migrantes procediendo ellas mismas de familias problemáticas y de lugares alejados que han tenido episodios graves de violencia política.

4. El problema de los niños, niñas y adolescentes desprotegidos es expresión histórica de ausencia paterna. Se cree que los hijos son de la mujer y la sociedad es muy permisiva ante el abandono del padre; más no así con el abandono materno.

5. Bunk aseveraba que poseería competencia profesional quien dispusiera de los conocimientos, destrezas y aptitudes necesarios para ejercer una profesión; pudiera resolver los problemas profesionales de forma autónoma y flexible y estuviera capacitado para colaborar en su entorno profesional y en la organización del trabajo.

6. Teniendo en cuenta las competencias profesionales se propone organizar equipos especializados de trabajadores sociales en las UPE con el objetivo de abordar problemas específicos o de trabajar con personas con determinadas características. Así pues, tendremos trabajadores sociales que conformen parte de equipos especializados en: deserción escolar en niños y adolescentes, niños con problemas conductuales, niños diagnosticados con problemas de salud mental, niños con discapacidades físicas o de aprendizaje, educación social y atención a la infancia, orientación y promoción familiar. El resultado: mayor eficiencia y rapidez en la atención.

7. En relación a la considerable generación de información, base de datos derivados de los expedientes que manejan los equipos multidisciplinarios de las UPE sobre la problemática de los niños y adolescentes sin cuidados parentales, es necesario señalar la importancia de la sistematización de experiencias del trabajador social para poder llegar a una interpretación crítica y analítica de una determinada parte de la realidad social. Resultado: producción de conocimientos de un segmento poblacional y aprendizaje colectivo de las colegas y demás profesionales.

\section{Bibliografía}

Ansion, J. y Tubino, F. (Ed.). (2007). Educar en ciudadanía intercultural: Experiencias y retos en la formación de estudiantes universitarios indígenas. Lima, Perú: Fondo Editorial de la Pontificia Universidad Católica del Perú. Bunk, G.P. (1994). La transmisión de las competencias en la formación y perfeccionamiento profesionales de la RFA (Asociación de Estudios sobre el Trabajo y la Organización de Empresas). Revista Europea de Formación Profesional, 1, pp. 8-14.

Defensoría del Pueblo (2011). Niños, niñasyadolescentesen abandono: aportes pana un nuevo modelo de atención. Serie Informes Defensoriales - Informe $N^{\circ} 153$. Primera edición: Lima, Perú: Autor.

Legis.pe (2018). Aprueban Reglamento del D.L. 1297, para la protección de niñas, niños y adolescentes sin cuidados parentales. Recuperado de: https://legis.pe/ aprueban-reglamento-d-1-1297-proteccion-ninos-adolescentes-cuidados-parentales/

Ministerio de la Mujer y Poblaciones Vulnerables (2013). Código de los Niños y Adolescentes. Recuperado de: https://www.mimp.gob.pe/files/direcciones/dgnna/Codigo_Ninos_Adolescentes.pdf

Ministerio de Economía y Finanzas, Ministerio de la Mujer y Poblaciones Vulnerables (2015). Criterios Técnicos Meta 20: Atención de Casos e Intervención de la Defensoría Municipal del Niño y del Adolescente DEMUNA en los Casos de Niñas, Niños y Adolescentes en Presunto Estado de Abandono. Recuperado de: https://www.mef.gob.pe/contenidos/presu_publ/migl/ municipalidades_pmm_pi/meta_20_Guia_metodologica.pdf

Muñoz, I. (Ed.). (2014). VII Seminario de Reforma del Estado. Inclusión Social: Enfoques, Políticas y Gestión Pública en el Perú. Lima, Perú: Fondo Editorial de la Pontificia Universidad Católica del Perú.

El Peruano (21 de julio de 2016). Decreto Supremo No 005-2016 - Aprueban el Reglamento del Servicio de Investigación Tutelar. Recuperado de https://cdn.www. gob.pe/uploads/document/file/30169/ds_005_2016_ mimp.df

Tapia, G. (2009). Un enfoque sobre la Investigación Tutelar Administrativa y el Sistema Único e Integral de Atención a Niños, Niñas y Adolescentes en Situación de Abandono. En: Primer Concurso Nacional de Ensayos Jurídicos. Trabajos ganadores. Recuperado de: http://repositorio.amag.edu.pe/bitstream/handle/123456789/128/Libro\%20Primer\%20 Concurso\%20 Nacional\%20de\%20Ensayos\%20 Juridicos.pdf?sequence=1 \&isAllowed $=y$ 
UNICEF (2006). Convención sobre los Derechos del Niño: 60 años unidos por la infancia 1946-2006. Recuperado de: https://www.un.org/es/events/childrenday/pdf/derechos.pdf

\section{ENCUESTA}

\section{APLICADA A LAS TRABAJADORAS SOCIALES DE LA DIRECCIÓN DE INVESTIGACIÓN TUTELAR}

La presente encuesta es el principal Instrumento de recolección de información aplicado a trabajadoras sociales que laboran en las instancias de Investigación Tutelar MIMP para investigar el aporte social en la optimización de casos de los niños en presunto estado de abandono. Por ello agradecemos de antemano sus respuestas las que serán de incalculable valor.

Fecha $\mathrm{N}^{\circ}$ de Encuesta.

Nombres y Apellidos

(Opcional)

\section{IDENTIFICACION}

1. Tiempo de servicio de la Trabajadora Social en la Dirección de Investigación Tutelar (DIT):

De 1 a 2 años( )

B) De 3 a 4 años( )

C) De 5 años a más( )

2. Lugar donde trabajó antes:

A) Sector público

a) Educación( ) b) Salud( ) c) CEM( ) d) DEMUNA( ) e)Yachay( ) e)Otros

B) Sector privado

a) Empresa( ) b) ONG( ) c) Iglesia( ) d) Organización comunal( ) e) Otros....

\section{DESCRIPCION DEL TRABAJO QUE REALIZA}

3. ¿Qué personas son las que más acuden a las entrevistas en los casos de presunto estado de abandono del Niño, Niña o Adolescente (NNA)? MARQUE CADA ALTERNATIVA CON UN NÚMERO DEL 1 AL 3, SIENDO:

POCO FRECUENTE (1)

REGULAR FRECUENCIA (2)

MAYOR FRECUENCIA (3)

a) La mamá( ) b) el papá( ) c) Ambos padres( )

d) Un hermano( ) e) Abuela materna( ) f) Abuela paterna( )

g) Padrinos() h) Vecinos( )

i) Representante de una institución( )

4. ¿ंA qué autoridad local recurre usted cuando llega al distrito donde vive la familia del NNA?

5. ¿Qué explicación da la madre o familiar sobre las causas de presunto estado de abandono de su hijo o hija?

6. En las visitas domiciliarias del último mes constata que los NNA viven con 


\section{MARQUE CADA ALTERNATIVA CON UN NÚMERO DEL 1 AL 3, SIENDO:}

POCO FRECUENTE (1)

REGULAR FRECUENCIA (2)

MAYOR FRECUENCIA (3)

a) La madre y el padre ( )

b) Sólo con uno de ellos ( )

c) Vive la madre con otra pareja y los hijos que tiene con éste ( )

d) Vive la madre con sus hijos y familiares cercanos ( )

e) Otros

7. ¿Cuánto tiempo la familia del NNA está viviendo en esa casa o cuarto²?

a) Menos de 1 año ( ) b) De 1 a 2 años ( ) c) Más de 3 años ( )

8. ¿Dónde vivían antes de llegar a ese lugar ${ }^{3}$ ?

a) En provincia ( ) b) En casa de familiares ( ) c) Otro distrito de Lima ( )

d) Otro

9. ¿En qué trabaja la familia del NNA en presunto estado de abandono?

a) Trabajo de la madre.

b) Trabajo del padre

c) otros familiares

10. Señale según usted algunas causas de presunto abandono infantil y adolescente. Escríbalas en orden de prioridad.
a)
b)
c)
d)

\section{INTERVENCION DEL TRABAJADOR SOCIAL}

11. ¿Cuáles son las 3 funciones más importantes que realiza como TRABAJADOR SOCIAL en la DIT?

a).

b)

c)

12. ¿Cuáles son los problemas más importantes que se presentan en la Investigación Tutelar?

a) Con las familias

b) Con el equipo

c) Con la parte administrativa

d) Otros

13. ¿Qué acciones o estrategias aplica cuando surgen estos problemas?

a) Con las familias

b) Con el equipo

c) Con la parte administrativa

d) Otros.

14. Díganos tres normas o leyes de Investigación Tutelar que le son más útiles?

2 Según el tiempo de residencia las redes sociales son más fuertes y los vecinos del lugar nos puede dar más información

3 Si son migrantes de primera generación o tienen familiares en la ciudad es posible rehabilitar las redes familiares más cercanas 
a)

b)

c)

15. ¿Qué logros ha tenido como trabajador social de la DIT?

a)

b)

c)

16. ¿Qué limitaciones y/o dificultades ha tenido como trabajador social de la DIT?

a)

b)

c)

17. ¿Qué acciones sugiere para disminuir las dificultades existentes en su trabajo en la DIT?

a)

b)

c)

18. ¿En qué acciones o espacios de intervención se articula más con los otros profesionales del equipo de la DIT?

a)

b)

c)

19. ¿Cuáles son las competencias que debe tener un trabajador social en la investigación tutelar?

a)

b)

c)

20. ¿Quiere decirnos algo respecto a la encuesta? ¿Faltó preguntar algo importante sobre el tema? ¿Tiene alguna sugerencia escríbala por favor?

GRACIAS 


\section{Contenidos de las competencias que conforman la competencia de acción.}

\section{Competencia técnica}

-Continuidad-

Conocimientos, destrezas, aptitudes

transciende los límites de la profesión

relacionada con la profesión

profundiza la profesión

amplía la profesión

relacionada con la empresa

\section{Competencia social} -Sociabilidad-

Formas de comportamiento

individuales: disposición al trabajo

capacidad de adaptación

capacidad de intervención

interpersonales: disposición a la cooperación

honradez

rectitud

altruismo

espíritu de equipo

\section{Competencia metodológica}

-Flexibilidad-

\section{Procedimientos}

procedimiento de trabajo variable

solución adaptada a la situación

resolución de problemas

pensamiento, trabajo, planificación, realización y control autónomos

capacidad de adaptación

\section{Competencia participativa}

-Participación-

Formas de organización

capacidad de coordinación

capacidad de organización

capacidad de relación

capacidad de convicción

capacidad de decisión

capacidad de responsabilidad

capacidad de dirección 